\title{
[gw22-e0834] FORECASTING LETHAL CARDIAC END POINTS OF HEART FAILURE PATIENTS WITH LOW EJECTION FRACTION USING REFINED MULTISCALE ENTROPY ANALYSIS
}

Nandor Balogh, ${ }^{1}$ Sandor Khoor, ${ }^{2}$ Mate Khoor, ${ }^{3}$ Katalin Fugedi, ${ }^{3}$ Ildiko Simon, ${ }^{3}$ Pal Kern, ${ }^{4}$ Gusztav Florian, ${ }^{3}$ Albert Kocsis, ${ }^{3}$ Ilona Kovacs ${ }^{3}$ Corpusnet Foundation for Computerisation; ${ }^{2}$ Medartintech Budapest; ${ }^{3}$ St Istvan Hospital Budapest; ${ }^{4}$ Kern Budapest

\subsection{6/heartjnl-2011-300867.636}

Purpose The aim of the study was to determine the risk of mortality, predicting by the Refined Multiscale Entropy (RMSE) analysis of heart failure patients with ejection fraction lower than 0.35 .

Method Refined Multi-Scale Entropy (RMSE) was proposed as a refinement of MSE, since it offers a way to solve two shortcomings that produce the MSE dependence on variance and on the shape of the power spectrum of the considered series. MSE is based on three steps: 1) progressive elimination of the fast time scales; 2) coarse graining procedure necessary to assess entropy rate; 3) calculation of the entropy rate. 168 patients

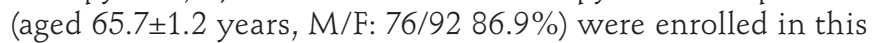
study. Patients were followed for 36 months. $24 \mathrm{~h}$ Holter registrations were performed in every 2 weeks. The high risk group (HRG) contains 27 patients, who had either sudden cardiac death, or death due to progressive heart failure, while the low risk group (LRG) contains 141 alive patients. Beside the descriptive statistics of the entropy values at the different scales, a multivariate discriminant model was developed, where the input variables were the change of the individual entropy/scale in time (72 Holter measurement for each patient), the output parameters were death (HRG) or alive (LRG).

Results All RMSE curves had a minimum at short time scale, followed by an exponential increment with t. Risk groups showed significant differences over a large interval of scales $(t=4-15)$, being the entropy-based complexity smaller in HRG than in LRG group. These results indicate a reduction of the HRV complexity in patients of death group. The largest statistical differentiation was obtained at the time scale $\mathrm{t}=8$ with a $\mathrm{p}$-value $=0.0024$ (LRG: $\mathrm{SE}=1.312 \pm 0.015$ and HRG: $\mathrm{SE}=1.107 \pm 0.022)$. The Wilks' statistic showed an excellent prediction (Wilks' $\lambda$ was $0.002 \mathrm{p}<0.0001$ ), and the multivariate discriminant score (DS_HRV) was calculated. The risk of death increased continuously with each quartile of DS_HRV with an adjusted RR of 4.2. A 3.5-fold increase in mortality in patients in the upper quartile compared to the lower quartile of DS_HRV (95\% CI 2.1 to 7.2, p<0.0001) was observed. 